\title{
Multiple solutions for a $p$-biharmonic equation with nonlinear boundary conditions
}

\author{
Wen-Wu Pan*, Xu-Dong Lin \\ Department of Science, Sichuan University of Science and Engineering, Zigong 643000, China \\ *Corresponding author, e-mail: wenwupan@sohu.com
}

Received 18 Feb 2014

Accepted 16 Apr 2015

ABSTRACT: In this paper, we obtain a multiplicity result for the p-biharmonic equation with smooth boundary.

KEYWORDS: critical point, variational method

MSC2010: 35J35 35J60 47J30 58E05

\section{INTRODUCTION AND PRELIMINARIES}

Let $\Omega$ be a bounded domain in $\mathbb{R}^{N}, N \geqslant 3$ with smooth boundary $\partial \Omega$ and a constant $p$ with $1<p<$ $N / 2$. In this paper, we consider the $p$-biharmonic equation

$$
\begin{cases}\Delta_{p}^{2} u+|u|^{p-2} u=\lambda f(u), & \text { in } \Omega, \\ \frac{\partial\left(|\Delta u|^{p-2} \Delta u\right)}{\partial v}=\mu g(u), & \text { on } \partial \Omega .\end{cases}
$$

The fourth-order equation with nonlinearity furnishes a model to study travelling waves in suspension bridges. Lazer and McKenna ${ }^{1}$ give a survey of results in this direction. This fourth-order semilinear elliptic problem can be considered as an analogue of a class of second-order problems which have been studied by many authors (see Refs. 2-4 and references therein). Bonder and Rossi ${ }^{5}$ study the existence of nontrivial solutions of the following fourth-order problem with nonlinear boundary conditions:

$$
\left\{\begin{array}{llrl}
-\Delta^{2} u & =u, & & \text { in } \Omega, \\
-\frac{\partial \Delta u}{\partial v}=f(x, u), & & \text { on } \partial \Omega .
\end{array}\right.
$$

They also impose one of the following boundary conditions: $\Delta u=0$ on $\partial \Omega$, or $\partial u / \partial v=0$ on $\partial \Omega$. The authors find infinitely many weak solutions for the above problems under suitable assumptions on the nonlinearity of $f$. The more general $p$-biharmonic equation has been considered in Refs. 2, 3. Differential equations with nonlinear boundary conditions have been considered by many authors in the last twenty years ${ }^{6}$.

Motivated by Refs. 2-4, 7, we show that problem (1) has at least two nontrivial solutions provided that $\lambda$ and $\mu$ are suitable. More precisely, we are interested in the following case: the functions $f, g$ are $(p-1)$-sublinear at infinity. Our main ingredient is a recent critical point result due to Bonanno $^{8}$.

In order to state our main result we introduce some hypotheses. We assume that the functions $f$ and $g: \mathbb{R} \rightarrow \mathbb{R}$ satisfy the following conditions.

(H1) There exist constants $C_{1}, C_{2}>0$ such that for all $t \in \mathbb{R}^{N}$,

$$
|f(t)| \leqslant C_{1}\left(1+|t|^{p-1}\right), \quad|g(t)| \leqslant C_{2}|t|^{p-1} .
$$

(H2) $f$ is superlinear at zero, i.e.,

$$
\lim _{t \rightarrow 0} \frac{f(t)}{|t|^{p-1}}=0 \text {. }
$$

(H3) If we set $F(t)=\int_{0}^{t} f(s) \mathrm{d} s$ and $G(t)=$ $\int_{0}^{t} g(s) \mathrm{d} s$, then there exists $t_{0} \in \mathbb{R}$ such that

$$
F\left(t_{0}\right)=\int_{0}^{t_{0}} f(s) \mathrm{d} s>0
$$

or

$$
G\left(t_{0}\right)=\int_{0}^{t_{0}} g(s) \mathrm{d} s>0 .
$$

Let $W^{2, p}(\Omega)$ be the usual Sobolev space with respect to the norm

$$
\|u\|_{2, p}^{p}=\int_{\Omega}\left(|\Delta u|^{p}+|u|^{p}\right) \mathrm{d} x
$$

which is equivalent to the standard norm and $W_{0}^{2, p}(\Omega)$ which is the closure of $C_{0}^{\infty}(\Omega)$ in $W^{2, p}(\Omega)$. 
Proposition 1 (see Ref. 7) For any $1<p<N / 2$ and $1 \leqslant q \leqslant p^{\star}=N p /(N-2 p)$, we denote by $S_{q, \Omega}$ the best constant in the embedding $W^{2, p}(\Omega) \hookrightarrow L^{q}(\Omega)$ and for all $1 \leqslant q \leqslant p_{\star}=(N-1) p /(N-2 p)$, we also denote by $S_{q, \partial \Omega}$ the best constant in the embedding $W^{2, p}(\Omega) \hookrightarrow L^{q}(\partial \Omega)$, i.e.,

$$
S_{q, \partial \Omega}=\inf _{u \in W^{2, p}(\Omega) \backslash W_{0}^{2, p}(\Omega)} \frac{\int_{\Omega}\left(|\Delta u|^{p}+|u|^{p}\right) \mathrm{d} x}{\left(\int_{\partial \Omega}|u|^{q} \mathrm{~d} \sigma\right)^{p / q}} .
$$

Moreover, if $1 \leqslant q<p^{\star}$, then the embedding $W^{2, p}(\Omega) \hookrightarrow L^{q}(\Omega)$ is compact and if $1 \leqslant q<p_{\star}$, then the embedding $W^{2, p}(\Omega) \hookrightarrow L^{q}(\partial \Omega)$ is compact.

Definition 1 We say that $u \in W^{2, p}(\Omega)$ is a weak solution of problem (1) if and only if

$$
\begin{array}{r}
\int_{\Omega}\left(|\Delta u|^{p-2} \Delta u \Delta \varphi+|u|^{p-2} u \varphi\right) \mathrm{d} x-\lambda \int_{\Omega} f(u) \varphi \mathrm{d} x \\
-\mu \int_{\partial \Omega} g(u) \varphi \mathrm{d} \sigma=0
\end{array}
$$

for all $\varphi \in W^{2, p}(\Omega)$.

Theorem 1 Assuming hypotheses (H1)-(H3) are fulfilled then there exist an open interval $\Lambda_{\mu}$ and a constant $\delta_{\mu}>0$ such that for all $\lambda \in \Lambda_{\mu}$, problem (1) has at least two weak solutions in $W^{2, p}(\Omega)$ whose $\|\cdot\|_{2, p}$-norms are less than $\delta_{\mu}$.

We emphasize that the condition (H3) cannot be omitted. Indeed, if $f \equiv 0$ and $g \equiv 0$, then (H1) and (H2) clearly hold, but problem (1) has only the trivial solution. Theorem 1 will be proved by using a result on the existence of at least three critical points by Bonanno ${ }^{8}$ which is a refinement of a general principle of Ricceri ${ }^{9,10}$. For the reader's convenience, we describe it as follows.

Theorem 2 (see Ref. 8) Let $(X,\|\cdot\|)$ be a separable and reflexive real Banach space, and $\Phi, \Psi: X \rightarrow \mathbb{R}$ be two continuously Gâteaux differentiable functionals. Assume that there exists $x_{0} \in X$ such that $\Phi\left(x_{0}\right)=$ $\Psi\left(x_{0}\right)=0, \Phi(x) \geqslant 0$ for all $x \in X$ and there exist $x_{1} \in X, \rho>0$ such that

(i) $\rho<\Phi\left(x_{1}\right)$,

(ii) $\sup _{\{\Phi(x)<\rho\}} \Psi(x)<\rho \Psi\left(x_{1}\right) / \Phi\left(x_{1}\right)$.

Further, put

$$
\bar{a}=\frac{\xi \rho}{\rho \frac{\Psi\left(x_{1}\right)}{\Phi\left(x_{1}\right)}-\sup _{\{\Phi(x)<\rho\}} \Psi(x)},
$$

with $\xi>1$, and assume that the functional $\Phi-\lambda \Psi$ is sequentially weakly lower semicontinuous, satisfies the Palais-Smale condition, and (iii) $\lim _{\|x\| \rightarrow \infty}[\Phi(x)-\lambda \Psi(x)]=+\infty$ for every $\lambda \in$ $[0, \bar{a}]$.

Then, there exist an open interval $\Lambda \subset[0, \bar{a}]$ and $a$ positive real number $\delta$ such that for each $\lambda \in \Lambda$, the equation $\Phi^{\prime}(u)-\lambda \Psi^{\prime}(u)=0$ has at least three solutions in $X$ whose norms are less than $\delta$.

\section{PROOF OF THEOREM 1}

For $\lambda$ and $\mu \in \mathbb{R}$, we define the functional $I_{\mu, \lambda}$ : $W^{2, p}(\Omega) \rightarrow \mathbb{R}$ by

$$
I_{\mu, \lambda}(u)=\Phi(u)-\lambda \Psi(u) \text { for all } u \in W^{2, p}(\Omega),
$$

where

$$
\begin{aligned}
& \Phi(u)=\int_{\Omega}\left(|\Delta u|^{p}+|u|^{p}\right) \mathrm{d} x-\mu \int_{\partial \Omega} G(u) \mathrm{d} \sigma, \\
& \Psi(u)=\int_{\Omega} F(u) \mathrm{d} x
\end{aligned}
$$

with $F(t)=\int_{0}^{t} f(t) \mathrm{d} t$ and $G(t)=\int_{0}^{t} g(t) \mathrm{d} t$. A simple computation implies that the functional $I_{\mu, \lambda}$ is $C^{1}$ and hence weak solutions of (1) correspond to the critical points of $I_{\mu, \lambda}$. We now check all assumptions of Theorem 2. For each $\mu \in\left[0, p S_{p, \partial \Omega} / C_{2}\right)$ we have $\Phi(u) \geqslant 0$ for all $u \in W^{2, p}(\Omega)$ and $\Phi(0)=\Psi(0)=0$ since the assumption (H1) holds. Moreover, by the compact embeddings $W^{2, p}(\Omega) \hookrightarrow L^{p}(\Omega)$ and $W^{2, p}(\Omega) \hookrightarrow L^{p}(\partial \Omega)$, a simple computation helps us to obtain the following lemma.

Lemma 1 For every $\mu \in\left[0, p S_{p, \partial \Omega} / C_{2}\right)$ and all $\lambda \in$ $\mathbb{R}$, the functional $I_{\mu, \lambda}$ is sequentially weakly lower semicontinuous on $W^{2, p}(\Omega)$.

Lemma 2 There exist two positive constants $\bar{\mu}$ and $\bar{\lambda}$ such that for all $\mu \in[0, \bar{\mu})$ and all $\lambda \in[0, \bar{\lambda})$, the functional $I_{\lambda, \mu}$ is coercive and satisfies the PalaisSmale condition in $W^{2, p}(\Omega)$.

Proof: By (H1), we have

$$
\begin{gathered}
I_{\mu, \lambda}(u)=\int_{\Omega}\left(|\Delta u|^{p}+|u|^{p}\right) \mathrm{d} x-\lambda \int_{\Omega} F(u) \mathrm{d} x \\
\quad-\mu \int_{\partial \Omega} G(u) \mathrm{d} \sigma \\
\geqslant\|u\|_{2, p}^{p}-\lambda C_{1} \int_{\Omega}\left(|u|+\frac{|u|^{p}}{p}\right) \mathrm{d} x \\
\quad-\mu \frac{C_{2}}{p} \int_{\partial \Omega}|u|^{p} \mathrm{~d} \sigma
\end{gathered}
$$




$$
\begin{aligned}
\geqslant\|u\|_{2, p}^{p} & \left(1-\lambda \frac{C_{1}}{p S_{p, \Omega}}-\mu \frac{C_{2}}{p S_{p, \partial \Omega}}\right) \\
& -\lambda \frac{C_{1}}{S_{1, \Omega}}\|u\|_{2, p} .
\end{aligned}
$$

Since relation (4) holds, by choosing

$$
\bar{\mu}=\bar{\lambda}=\min \left\{\frac{p S_{p, \Omega}}{2 C_{1}}, \frac{p S_{p, \partial \Omega}}{2 C_{2}}\right\},
$$

where $C_{1}, C_{2}$ are given in (H1), we conclude that for all $\lambda \in[0, \bar{\lambda})$ and all $\mu \in[0, \bar{\mu})$, the functional $I_{\mu, \lambda}$ is coercive.

Now, let $\left\{u_{n}\right\}$ be a Palais-Smale sequence for the functional $I_{\mu, \lambda}$ in $W^{2, p}(\Omega)$, i.e.,

$$
\left|I_{\mu, \lambda}\left(u_{n}\right)\right| \leqslant \bar{c}, \quad I_{\mu, \lambda}^{\prime}\left(u_{n}\right) \rightarrow 0 \text { in } W^{-2, p}(\Omega),
$$

where $\bar{c}$ is a constant and $W^{-2, p}(\Omega)$ is the dual space of $W^{2, p}(\Omega)$. Since $I_{\mu, \lambda}$ is coercive, the sequence $\left\{u_{n}\right\}$ is bounded in $W^{2, p}(\Omega)$. Hence there exists a subsequence of $\left\{u_{n}\right\}$, still denoted by $\left\{u_{n}\right\}$ such that $\left\{u_{n}\right\}$ converges weakly to some $u \in W^{2, p}(\Omega)$ and hence converges strongly to $u$ in $L^{p}(\Omega)$ and in $L^{p}(\partial \Omega)$. We shall prove that $\left\{u_{n}\right\}$ converges strongly to $u$ in $W^{2, p}(\Omega)$. Indeed, we have

$$
\begin{aligned}
& \left\|u_{n}-u\right\|_{2, p}^{p} \\
& \leqslant \int_{\Omega}\left(\left|\Delta u_{n}\right|^{p-2} \Delta u_{n}-|\Delta u|^{p-2} \Delta u\right) \\
& \quad \times\left(\Delta u_{n}-\Delta u\right) \mathrm{d} x \\
& \quad+\int_{\Omega}\left(\left|u_{n}\right|^{p-2} u_{n}-|u|^{p-2} u\right)\left(u_{n}-u\right) \mathrm{d} x \\
& =\left[I_{\mu, \lambda}^{\prime}\left(u_{n}\right)-I_{\mu, \lambda}^{\prime}(u)\right]\left(u_{n}-u\right) \\
& \quad+\lambda \int_{\Omega}\left[f\left(u_{n}\right)-f(u)\right]\left(u_{n}-u\right) \mathrm{d} x \\
& \quad+\mu \int_{\partial \Omega}\left[g\left(u_{n}\right)-g(u)\right]\left(u_{n}-u\right) \mathrm{d} x .
\end{aligned}
$$

On the other hand, the compact embeddings and (H1) imply

$$
\begin{aligned}
& \left|\int_{\Omega}\left[f\left(u_{n}\right)-f(u)\right]\left(u_{n}-u\right) \mathrm{d} x\right| \\
& \leqslant \int_{\Omega}\left|f\left(u_{n}\right)-f(u)\right|\left|u_{n}-u\right| \mathrm{d} x \\
& \leqslant C_{1} \int_{\Omega}\left(2+\left|u_{n}\right|^{p-1}+|u|^{p-1}\right)\left|u_{n}-u\right| \mathrm{d} x \\
& \leqslant C_{1}\left(2 \operatorname{meas}(\Omega)^{(p-1) / p}+\left\|u_{n}\right\|_{L^{p}(\Omega)}^{p-1}+\|u\|_{L^{p}(\Omega)}^{p-1}\right) \\
& \quad \times\left\|u_{n}-u\right\|_{L^{p}(\Omega)}^{p},
\end{aligned}
$$

where meas $(\Omega)$ denotes the Lebesgue measure of $\Omega$, which approaches 0 as $n \rightarrow \infty$. Similarly, we obtain

$$
\begin{aligned}
& \left|\int_{\partial \Omega}\left[g\left(u_{n}\right)-g(u)\right]\left(u_{n}-u\right) \mathrm{d} x\right| \\
& \quad \leqslant \int_{\partial \Omega}\left|g\left(u_{n}\right)-g(u)\right|\left|u_{n}-u\right| \mathrm{d} x \\
& \quad \leqslant C_{2} \int_{\partial \Omega}\left(\left|u_{n}\right|^{p-1}+|u|^{p-1}\right)\left|u_{n}-u\right| \mathrm{d} x \\
& \quad \leqslant C_{2}\left(\left\|u_{n}\right\|_{L^{p}(\partial \Omega)}^{p-1}+\|u\|_{L^{p}(\partial \Omega)}^{p-1}\right)\left\|u_{n}-u\right\|_{L^{p}(\partial \Omega)}^{p}
\end{aligned}
$$

which approaches 0 as $n \rightarrow \infty$. Hence by (5) we have $\left\|u_{n}-u\right\|_{2, p} \rightarrow 0$ as $n \rightarrow \infty$.

Lemma 3 For every $\mu \in[0, \bar{\mu})$ with $\bar{\mu}$ as in Lemma 2 , we have

$$
\lim _{\rho \rightarrow 0^{+}} \frac{\sup \{\Psi(u): \Phi(u)<\rho\}}{\rho}=0 .
$$

Proof: Let $\lambda \in[0, \bar{\lambda})$ and $\mu \in[0, \bar{\mu})$ be fixed. By (H2), for any $\varepsilon>0$, there exists $\delta=\delta(\varepsilon)>0$ such that

$$
|f(s)|<\varepsilon p S_{p, \Omega}\left(1-\mu \frac{C_{2}}{p S_{p, \partial \Omega}}\right)|s|^{p-1} \forall|s|<\delta .
$$

We first fix $q \in\left(p, p^{\star}\right)$. Combining the above inequalities with (H1) we deduce that

$$
|F(s)| \leqslant \varepsilon S_{p, \Omega}\left(1-\mu \frac{C_{2}}{p S_{p, \partial \Omega}}\right)|s|^{p}+C_{\delta}|s|^{q},
$$

for all $s \in \mathbb{R}$, where $C_{\delta}$ is a constant depending on $\delta$. Now, for every $\rho>0$, we define the sets

$$
\mathscr{B}_{\rho}^{1}=\left\{u \in W^{2, p}(\Omega): \Phi(u)<\rho\right\}
$$

and

$$
\mathscr{B}_{\rho}^{2}=\left\{u \in W^{2, p}(\Omega):\left(1-\mu \frac{C_{2}}{p S_{p, \partial \Omega}}\right)\|u\|_{2, p}^{p}<\rho\right\} .
$$

Then $\mathscr{B}_{\rho}^{1} \subset \mathscr{B}_{\rho}^{2}$. From (6) we get

$$
|\Psi(u)| \leqslant \varepsilon\left(1-\mu \frac{C_{2}}{p S_{p, \partial \Omega}}\right)\|u\|_{2, p}^{p}+\frac{C_{\delta}}{S_{q, \Omega}^{q / p}}\|u\|_{2, p}^{q} .
$$

It is clear that $0 \in \mathscr{B}_{\rho}^{1}$ and $\Psi(0)=0$. Hence, $0 \leqslant$ $\sup _{u \in \mathscr{B}_{\rho}^{1}} \Psi(u)$. Using (7) we get

$$
\begin{aligned}
0 & \leqslant \frac{\sup _{u \in \mathscr{B}_{\rho}^{1}} \Psi(u)}{\rho} \leqslant \frac{\sup _{u \in \mathscr{B}_{\rho}^{2}} \Psi(u)}{\rho} \\
& \leqslant \varepsilon+\frac{C_{\delta}}{S_{q, \Omega}^{q / p}}\left(1-\mu \frac{C_{2}}{p S_{p, \partial \Omega}}\right)^{-q / p} \rho^{q / p-1} .
\end{aligned}
$$


We complete the proof of the lemma by letting $\rho \rightarrow$ $0^{+}$, since $\varepsilon>0$ is arbitrary.

Proof of Theorem 1: Let $s_{0}$ be as in (H3). We choose a constant $r_{0}>0$ such that $r_{0}<\operatorname{dist}(0, \partial \Omega)$. For each $\sigma \in(0,1)$ we define the function $u_{\sigma}(x)=0$, if $x \in \mathbb{R}^{N} \backslash B_{r_{0}}(0), u_{\sigma}(x)=s_{0}$, if $x \in B_{\sigma r_{0}}(0), u_{\sigma}(x)=$ $\frac{1}{2} s_{0} \sin \left[\left(\pi /(1-\sigma) r_{0}\right)\left(\frac{1}{2}(1+\sigma) r_{0}-\left|x-x_{0}\right|\right)\right]+\frac{1}{2} s_{0}$, if $x \in B_{r_{0}}(0) \backslash B_{\sigma r_{0}}(0)$, where $B_{r_{0}}(0)$ denotes the open ball with centre 0 and radius $r_{0}>0$. Then it is clear that $u_{\sigma} \in W^{2, p}(\Omega)$. We have that $u_{\sigma}(x) \in W^{2, p}$ and $\left|u_{\sigma}(x)\right| \leqslant s_{0}$ for all $x \in \mathbb{R}^{N}$. Moreover, we have

$$
\Psi\left(u_{\sigma}\right) \geqslant\left[F\left(s_{0}\right) \sigma^{N}-\max _{|t| \leqslant\left|s_{0}\right|}|F(t)|\left(1-\sigma^{N}\right)\right] \omega_{N} r_{0}^{N},
$$

where $\omega_{N}$ is the volume of the unit ball in $\mathbb{R}^{N}$. From (9), there is $\sigma_{0}>0$ such that $\left\|u_{\sigma_{0}}\right\|_{2, p}>0$ and $\Psi\left(u_{\sigma_{0}}\right)>0$. Now, by Lemma 3 , we can choose $\rho_{0} \in(0,1)$ such that

$$
\rho_{0}<\left(1-\mu \frac{C_{2}}{p S_{p, \partial \Omega}}\right)\left\|u_{\sigma_{0}}\right\|_{2, p}^{p} \leqslant \Phi\left(u_{\sigma_{0}}\right)
$$

and satisfies

$$
\frac{\sup _{\Phi(u)<\rho_{0}} \Psi(u)}{\rho_{0}}<\frac{\Psi\left(u_{\sigma_{0}}\right)}{2 \Phi\left(u_{\sigma_{0}}\right)} .
$$

To apply Theorem 2, we choose $x_{1}=u_{\sigma_{0}}$ and $x_{0}=0$. Then the assumptions (i) and (ii) of Theorem 2 are satisfied. Next, we define

$$
\begin{aligned}
& a_{\mu}=\frac{1+\rho_{0}}{\frac{\Psi\left(u_{\sigma_{0}}\right)}{\Phi\left(u_{\sigma_{0}}\right)}-\frac{\sup \left\{\Psi(u): \Phi(u)<\rho_{0}\right\}}{\rho_{0}}}>0, \\
& \bar{a}_{\mu}=\min \left\{a_{\mu}, \bar{\lambda}\right\} .
\end{aligned}
$$

and a simple computation implies that (iii) is verified. Hence, there exist an open interval $\Lambda_{\mu} \subset$ $\left[0, \bar{a}_{\mu}\right]$ and a real positive number $\delta_{\mu}$ such that for each $\lambda \in \Lambda_{\mu}$, the equation $I_{\mu, \lambda}^{\prime}(u)=\Phi_{\mu}^{\prime}(u)-$ $\lambda \Psi^{\prime}(u)=0$ has at least three solutions in $W^{2, p}(\Omega)$ whose norms are less than $\delta_{\mu}$. By (H1) and (H2), one of them may be the trivial one. Thus (1) has at least two weak solutions in $W^{2, p}(\Omega)$. The proof is complete.

Acknowledgements: The project was supported by the Scientific Research Fund of SUSE (No. 2011KY03) and the Scientific Research Fund of Sichuan Provincial Education Department (No. 12ZB081).

\section{REFERENCES}

1. Lazer AC, McKenna PJ (1990) Large-amplitude periodic oscillations in suspension bridges: some new connections with nonlinear analysis. SIAM Rev 32, 537-78.

2. Candito P, Li L, Livrea R (2012) Infinitely many solutions for a perturbed nonlinear Navier boundary value problem involving the $p$-biharmonic. Nonlin Anal 75, 6360-9.

3. Li L, Heidarkhani S (2012) Existence of three solutions to a double eigenvalue problem for the $p$ biharmonic equation. Ann Polon Math 104, 71-80.

4. Li L, Tang CL (2010) Existence of three solutions for ( $p, q)$-biharmonic systems. Nonlin Anal 73, 796-805.

5. Bonder JF, Rossi JD (2002) A fourth order elliptic equation with nonlinear boundary conditions. Nonlin Anal 49, 1037-47.

6. Rossi JD (2005) Elliptic problems with nonlinear boundary conditions and the Sobolev trace theorem. In: Chipot M, Quittner P (eds) Stationary Partial Differential Equations Vol II, Elsevier, Amsterdam, pp 311-406.

7. El Khalil A, Kellati S, Touzani A (2005) A nonlinear boundary problem involving the $p$-bilaplacian operator. Int J Math Math Sci 2005, 1525-37.

8. Bonanno G (2003) Some remarks on a three critical points theorem. Nonlin Anal 54, 651-65.

9. Ricceri B (2000) On a three critical points theorem. Arch Math 75, 220-6.

10. Ricceri B (2000) Existence of three solutions for a class of elliptic eigenvalue problems. Math Comput Model 32, 1485-94. 